\title{
Evaluating the Effects of Competition vs Collaboration on User Engagement in an Immersive Game using Natural Interaction
}

\author{
Brondi R., Avveduto G., Alem L., Faita C., Carrozzino M., Tecchia F., Pisan Y. and Bergamasco M. \\ Scuola Superiore Sant'Anna - University of Technology Sydney
}

\section{INTRODUCTION}

Social experience can deeply impact on gaming experience and is often used to increase enjoyment and retention. In the literature two main categories of social interaction can be identified: competition and collaboration. Player engagement has been widely studied under different conditions related to the type of social interaction taking place during the game. However, rich and newly available contexts based on emerging paradigms, such as those enabled by Natural User Interfaces, have not been yet extensively addressed. In the current study the impact of collaborative and competitive goal structures on player engagement, awareness and social presence is evaluated in the context of a jigsaw puzzle game taking place in a Shared Virtual Environment using a highly immersive setup exploiting natural user interaction.

\section{METHOD}

The experiment has adopted a within-pairs design. A total of 24 subjects (12 couples) have played two multi-player jigsaw puzzle games. The two games differ in terms of the implemented game mechanics only: competitive and collaborative. In order to provide a high immersion to the user and enable a natural interaction with the VE, a setup similar to [Tecchia et al. 2014] has been used. The user wearing a Head Mounted Display (HMD) is free to move naturally in a physical/virtual environment; the perception of the self and of the partner in the VE, and the natural interaction with the surrounding world, are enabled by depth cameras and inertial/visual tracking techniques (see Fig. ??). The participants are able to communicate both verbally and using their bodies (e.g. using gestures). Participants have been able to explore new and effective way of communication through the provided Natural User Interface. The game is played by two players at the same time. The participants, physically located in two different places, share the same VE during the game session. The captures of the users' bodies and the game's data (e.g. scores, tiles positions, etc.) are streamed between the two physical setups. A proxy for each player, made by a textured mesh reconstructed from the RGBD data, and a virtual head, which replicates user's movements, is shown in the VE. The player's goal playing the competitive game is to finish the puzzle before the time is over and correctly placing more tiles than the opponent. The aim of the collaborative game therefore consists in working together with the partner in order to solve the puzzle before the time is over.

Player engagement and social presence during the experimental

*e-mail:r.brondi@sssup.it

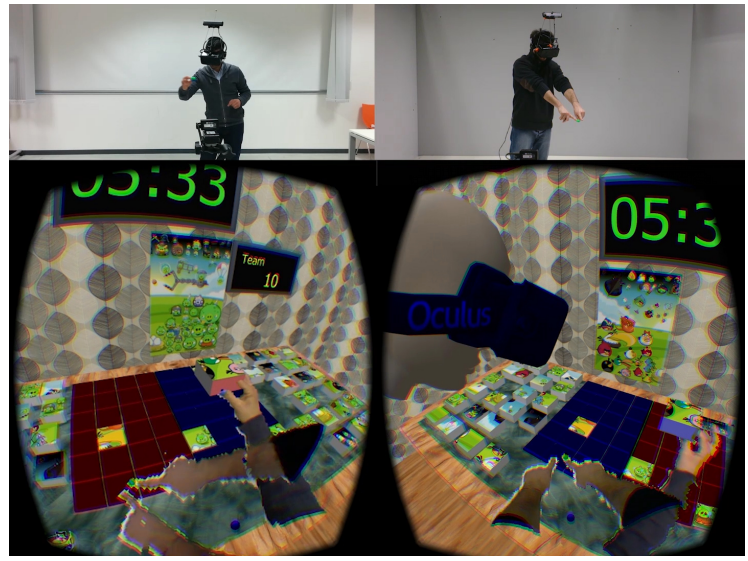

Figure 1: Snapshot of a running session. On each side the player and the image seen trough the Oculus are shown.

sessions have been assessed using a subset of Game Engagement Questionnaire - Competence, Flow, Tension/Annoyance, Challenge, Negative affect and Positive affect — and of Social Presence in Gaming Questionnaire - Empathy and Behavioural involvement. Objective measurements have been recorded through the games.

\section{FINDINGS}

Initial findings from our study suggest that the embodiment experience offered by the implemented technological solution positively affects both game mechanics. Collaborative games should be preferred when high levels of social presence and emotional connection are required. Collaboration allows also to improve the awareness of the partner and should be preferred when being conscious of what the other players are doing is important. According to previous research [Brondi et al. 2015], competition results to be the preferred paradigm when the improvement of user performances matters. Natural interaction has proven to be extremely powerful when an intense and effective communication is required. Particular attention must be paid when designing immersive Natural User Interfaces in order to compensate missing stimuli and improve awareness of the other in competitive games.

\section{References}

Brondi, R., Alem, L., Avveduto, G., Faita, C., Carrozzino, M., Tecchia, F., and Bergamasco, M. 2015. Evaluating the impact of highly immersive technologies and natural interaction on player engagement and flow experience in games. In Entertainment Computing-ICEC 2015. Springer.

Tecchia, F., Avveduto, G., Brondi, R., Carrozzino, M., BergamAsco, M., AND Alem, L. 2014. I'm in vr!: using your own hands in a fully immersive mr system. In Proceedings of the 20th ACM Symposium on Virtual Reality Software and Technology, ACM, 73-76. 